\title{
De novo assembly and characterization of the root transcriptome of Aegilops variabilis during an interaction with the cereal cyst nematode
}

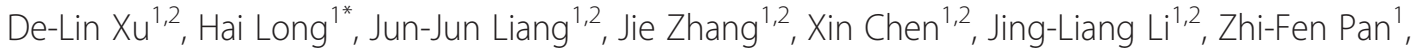
Guang-Bing Deng ${ }^{1}$ and Mao-Qun $\mathrm{Yu}^{{ }^{*}}$

\begin{abstract}
Background: Aegilops variabilis No.1 is highly resistant to cereal cyst nematode (CCN). However, a lack of genomic information has restricted studies on CCN resistance genes in Ae. variabilis and has limited genetic applications in wheat breeding.

Results: Using RNA-Seq technology, we generated a root transcriptome at a sequencing depth of 4.69 gigabases of Ae. variabilis No. 1 from a pooled RNA sample. The sample contained equal amounts of RNA extracted from CCN-infected and untreated control plants at three time-points. Using the Trinity method, nearly 52,081,238 high-quality trimmed reads were assembled into a non-redundant set of 118,064 unigenes with an average length of $500 \mathrm{bp}$ and an N50 of $599 \mathrm{bp}$. The total assembly was $59.09 \mathrm{Mb}$ of unique transcriptome sequences with average read-depth coverage of 33.25x. In BLAST searches of our database against public databases, $66.46 \%(78,467)$ of the unigenes were annotated with gene descriptions, conserved protein domains, or gene ontology terms. Functional categorization further revealed 7,408 individual unigenes and three pathways related to plant stress resistance.

Conclusions: We conducted high-resolution transcriptome profiling related to root development and the response to CCN infection in Ae. variabilis No.1. This research facilitates further studies on gene discovery and on the molecular mechanisms related to CCN resistance.
\end{abstract}

\section{Background}

Cereal cyst nematode (causal agent Heterodeta avenae) causes cereal disease in many regions of the world [1-5], and results in economic losses of billions of dollars annually [6]. Although CCNs have caused serious economic losses over the last 40 years [1], only a few $\mathrm{CCN}$ resistance genes have been genetically mapped on the genomes of wheat (Cre1 and $\mathrm{Cre} 8$ ) and its relatives, such as those in the genus Aegilops (Cre2-7), Secale cereale (CreR) (reviewed in Smiley and Nicol (2009) [7]) and Hordeum vulgare (Ha1-4) (reviewed in Bakker et al. (2006) [8]). The molecular mechanism of $\mathrm{CCN}$ resistance remains unknown.

Members of the genus Aegilops readily hybridize with bread wheat as the male parent [9]. Aegilops species are valuable genetic resources for breeding for disease

\footnotetext{
* Correspondence: hailong@cib.ac.cn; yumaoqun@cib.ac.cn

${ }^{1}$ Chengdu Institute of Biology, Chinese Academy of Sciences, Chengdu,

Sichuan, China

Full list of author information is available at the end of the article
}

resistance in wheat; for example, for resistance to Cochliobolus sativus (spot blotch), Tilletia indica (Karnal bunt), and powdery mildew [10,11]. Ae. variabilis accession No.1 $\left(2 \mathrm{n}=4 x=28\right.$, UUS $\left.^{\vee} S^{\vee}\right)$ (syn. Triticum peregrinum (Hack In J. Fraser) Marie \& Hackel) was reported to harbor resistance genes to both CCN and root knot nematode (Meloidogyne naasi) $[12,13]$. A greater understanding of the mechanism of CCN resistance in Ae. variabilis is necessary for wheat breeding. However, the major barrier against using genomic approaches to improve Ae. variabilis is that the genome sequence, cDNA libraries, EST databases, and microarray platform information are not available [14].

Recent developments in RNA-Seq technology have enabled very efficient probing of transcriptomic data [15-19]. This method not only detects transcripts that correspond to existing genomic sequences, but it can also be used for de novo assembly of short reads for gene discovery and expression profiling in organisms for which there is no reference genome [17,20-26].

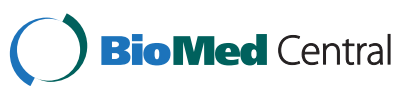


In the present study, we analyzed the root transcriptome of Ae. variabilis using RNA-Seq technology. We used two methods, SOAPdenovo and Trinity, for de novo assembly of the transcriptome, and compared their results. Characterization of the transcriptome data assembled by Trinity give a high-resolution insight into the genes involved in several major metabolic pathways associated with root development and plant defense. This research will serve as a public information platform for further studies on the evolution and function of genes in Ae. variabilis, and provides a thorough insight into the gene expression profiles associated with the response to $\mathrm{CCN}$ infection in Ae. variabilis.

\section{Methods}

\section{Plant material and pathogen infection}

Ae. variabilis accession No.1 was used for transcriptomic profiling of genes expressed in roots. Grains of Ae. variabilis No.1 were surface-sterilized in a solution containing $3 \%(\mathrm{v} / \mathrm{v})$ hypochlorite and $0.01 \%(\mathrm{v} / \mathrm{v})$ Tween 20 for $5 \mathrm{~min}$ and rinsed three times with sterile water [27]. The seeds were germinated in Petri dishes $(5-\mathrm{cm}$ diameter) on wet paper at $20 \mathrm{C}$ under a 16-h light/8-h dark photoperiod. After 10 days, seedlings were divided into two groups. One group was inoculated with 1,000 second-stage juveniles (J2) of CCN per plant, and the other group (negative control) was not inoculated with CCN $[28,29]$. Thirty hours after inoculation, the roots were thoroughly washed three times with sterile water (each $10 \mathrm{~min}$ ) to remove CCNs adhering to roots. Then, plants were transplanted into $500-\mathrm{ml}$ glass containers filled with sterilized perlite, and were grown at $20 \mathrm{C}$ under a 16-h light/8-h dark photoperiod. These conditions prevented further $\mathrm{CCN}$ penetration and ensured synchronized development of syncytia $[27,30]$.

\section{RNA isolation}

Successful CCN inoculation was confirmed by observing roots under a microscope (Additional file 1). Roots of $\mathrm{CCN}$-infected and non-infected plants were sampled at 30 hpi (hours post inoculation), 3 dpi (days post inoculation) and 9 dpi for RNA extraction [27,31,32]. Each sample consisted of 15 individuals. Total RNA was extracted with a Biomiga RNA kit according to the manufacturer's protocol (Biomiga, San Diego, CA, USA). The concentration and quality of each RNA sample was determined using a NanoDrop $2000^{\mathrm{TM}}$ micro-volume spectrophotometer (Thermo Scientific, Waltham, MA, USA). Equal amounts of total RNA from each sample were pooled to construct the cDNA library. Pooling is a cost-effective strategy when the primary research goal is to identify gene expression profiles. This strategy was well-justified based on statistical and practical considerations [33-35].
Construction of cDNA library and Illumina deep-sequencing The cDNA library was constructed using an mRNA-Seq assay for paired-end transcriptome sequencing. The library construction and sequencing were performed by the Beijing Genomics Institute (BGI)-Shenzhen, Shenzhen, China. Briefly, mRNA was enriched from $20 \mu \mathrm{g}$ total RNA using oligo dT magnetic beads, and was then cleaved into 200-700 nt fragments by incubation with RNA Fragmentation Reagent. The fragmented mRNA was converted into double-stranded cDNA by priming with random hexamerprimers, purified with a QiaQuick PCR extraction kit (QIAGEN Inc., Valencia, CA, USA), and then washed with EB buffer for end repairing and single nucleotide adenine addition. Finally, sequencing adaptors were ligated onto the fragments, and the required fragments were purified by agarose gel electrophoresis and enriched by PCR amplification to construct the cDNA library. The library was loaded onto the channels of an Illumina $\mathrm{HiSeq}^{\mathrm{TM}} 2000$ instrument for 4 gigabase in-depth sequencing, which was used to obtain more detailed information about gene expression. Each paired-end library had an insert size of 200-700 bp. The average read length of $90 \mathrm{bp}$ was generated as raw data. The data sets are available at the NCBI SRA database with the accession number of SRA050454.

\section{De novo assembly and sequence clustering}

The clean reads were obtained from raw data by filtering out adaptor-only reads, reads containing more than $5 \%$ unknown nucleotides, and low-quality reads (reads containing more than $50 \%$ bases with $\mathrm{Q}$-value $\leq 20$ ). Then $d e$ novo assembly of the clean reads was performed to generate non-redundant unigenes. We used two methods for de novo assembly; SOAPdenovo 63mer-V1.05 [36] with optimized $k$-mer length of 41 , and the Trinity method [19] with optimized $k$-mer length of 25 .

Sequence directions of the resulting unigenes were determined by performing BLASTX searches against protein databases, with the priority order of NR (nonredundant protein sequences in NCBI), Swiss-Prot, Kyoto Encyclopedia of Genes and Genomes database (KEGG), and COG (E-value $\leq 1 \mathrm{e}-5)$ if conflicting results were obtained. ESTScan software [37] was also used to determine the directions of sequences that were not aligned to those in any of the databases mentioned above.

The expression levels of unigenes were measured as the number of clean reads mapped to its sequence. The number of clean reads mapped to each annotated unigene was calculated and then normalized to RPKM (reads per Kb per million reads) with ERANGE3.1 software [18] and adjusted by a normalized factor [38].

\section{Functional categorization of unigenes}

The unigenes assembled by the Trinity method that were longer than $200 \mathrm{bp}$ were annotated according to their 
sequence similarity to previously annotated genes. We used sequence-based and domain alignments to compare sequences. Sequence-based alignments were performed against three public databases (NR, Swiss-Prot, and KEGG; significant thresholds of E-value $\leq 1 \mathrm{e}-5$ ). Domainbased alignments were carried out against the COG database at NCBI with a cut-off E-value of $\leq 1 \mathrm{e}-5$.

The resulting BLAST hits were processed by Blast2GO software [39] to retrieve associated Gene Ontology (GO) terms describing biological processes, molecular functions, and cellular components [40]. By using specific gene identifiers and accession numbers, Blast2GO produces GO annotations as well as corresponding enzyme commission numbers (EC) for sequences with an E-value $\leq 1 \mathrm{e}-5$.

KEGG mapping was used to determine the metabolic pathways $[41,42]$. The sequences with corresponding ECs obtained from Blast2GO were mapped to the KEGG metabolic pathway database. To further enrich the pathway annotation and to identify the BRITE functional hierarchies, sequences were also submitted to the KEGG Automatic Annotation Server (KAAS) [43], and the single-directional best hit information method was selected. KAAS annotates every submitted sequence with KEGG orthology (KO) identifiers, which represents an orthologous group of genes directly linked to an object in the KEGG pathways and BRITE functional hierarchy $[43,44]$. Therefore, these methods incorporate different types of relationships that exist in biological systems (i.e. genetic and environmental information processing, cellular processes, and organism systems).

\section{Results}

Transcriptome sequencing, de novo assembly, and sequence analysis

We constructed a cDNA library of pooled RNA samples to generate a transcriptomic view of genes expressed in the root of uninfected and $\mathrm{CCN}$-infected Ae variabilis. Approximately 4,687,311,420 base pairs of raw data were generated, yielding a total of $54,267,786$ clean reads that were $90 \mathrm{bp}$ in length (Table 1). Of the clean reads, 91.63\% had a Phred quality score of $\leq$ Q20 level (error probability of 0.01 ).

All trimmed reads were de novo assembled by SOAP denovo and Trinity programs (Table 1). SOAPdenovo

Table 1 Summary of de novo sequence assembly

\begin{tabular}{cccccc}
\hline & $\begin{array}{c}\text { Sequences } \\
(\mathbf{n})\end{array}$ & $\begin{array}{c}\text { Base pairs } \\
(\mathbf{M b p})\end{array}$ & $\begin{array}{c}\text { Length } \\
\text { range (bp) }\end{array}$ & $\begin{array}{c}\text { Mean } \\
\text { length (bp) }\end{array}$ & $\begin{array}{c}\text { N50 } \\
\text { (bp) }\end{array}$ \\
\hline Clean reads & $52,081,238$ & $4,687.31$ & $90-90$ & 90 & 90 \\
SOAP contigs & 336,641 & 60.21 & $60-3911$ & 200 & 229 \\
Trinity contigs & 481,672 & 92.50 & $75-3696$ & 192 & 250 \\
SOAP unigenes & 130,487 & 45.86 & $150-4113$ & 351 & 392 \\
Trinity unigenes & 118,064 & 59.09 & $200-4214$ & 500 & 599 \\
\hline
\end{tabular}

produced 336,641 contigs of 60 to 3,911 bp with an average length of $200 \mathrm{bp}$ and an N50 of 229 bp (i.e., $50 \%$ of the assembled bases were incorporated into contigs of 229 bp or longer). The majority of the contigs were shorter than $200 \mathrm{bp}$ (71.97\%), and 2,722 contigs (0.81\%) were longer than $1,000 \mathrm{bp}$. Trinity generated 481,672 contigs ranging from 75 to $3696 \mathrm{bp}$ with an average length of $192 \mathrm{bp}$ and an N50 of $250 \mathrm{bp}$. Similar to the SOAPdenovo assembly, most contigs were shorter than 200 bp $(79.65 \%)$ but there was a greater number of longer contigs-11,394 contigs (2.37\%) were longer than $1000 \mathrm{bp}$. The size distribution of these contigs is shown in Table 2. A total of 130,487 unigenes were further generated by SOAPdenovo. The unigenes had an average length of 351 bp and an N50 of 392 bp. Among the unigenes, 37,828 (28.99\%) were shorter than $200 \mathrm{bp}$ and $4,702(3.60 \%)$ were longer than $1,000 \mathrm{bp}$. The Trinity method generated fewer unigenes $(118,064)$. These unigenes had an average length of $500 \mathrm{bp}$ and an N50 of 599 bp. Among the unigenes, 64,330 unigenes (54.49\%) were 200 to $400 \mathrm{bp}$ in length. There were no unigenes shorter than $200 \mathrm{bp}$ and $9.00 \%(10,622)$ of all generated unigenes were longer than 1,000 bp (Table 2).

To assess the quality of the data set, we evaluated the assembled unigenes to determine the presence and length of gaps in the sequences. The analysis showed that $1.56 \%$ of the unigenes assembled by SOAPdenovo contained gaps, whereas those assembled by Trinity contained no gaps (Figure 1a).

Because there is no transcriptome profile of Ae. variabilis available for comparison, we used a web-based tool, ESTcal [45], to evaluate the depth and breadth of our data set. The read-depth coverage for $35.29 \%$ of SOAPdenovo-generated unigenes and for $22.79 \%$ of Trinitygenerated unigenes was greater than 20 fold (Figure 1b), with an average read-depth coverage of 33.54-fold and 33.25-fold, respectively.

Table 2 Length and number distribution of the unigenes and contigs

\begin{tabular}{cccccc}
\hline $\begin{array}{c}\text { Length } \\
\text { range (bp) }\end{array}$ & \multicolumn{2}{c}{ SOAPdenovo } & & \multicolumn{2}{c}{ Trinity } \\
\cline { 2 - 3 } \cline { 5 - 6 } \cline { 5 - 6 } & Contig No. & Unigene No. & & Contig No. & Unigene No. \\
\hline 200 & 242,275 & 37,828 & & 383,651 & 0 \\
$200-299$ & 51,635 & 42,043 & & 41,094 & 41,031 \\
$300-399$ & 19,366 & 19,062 & & 18,886 & 23,299 \\
$400-499$ & 9,163 & 10,457 & & 9,454 & 13,917 \\
$500-999$ & 11,480 & 16,395 & & 17,193 & 29,195 \\
$1000-1999$ & 2,497 & 4,120 & & 8,913 & 10,027 \\
$2000-3000$ & 208 & 505 & & 1969 & 572 \\
$>3000$ & 17 & 77 & & 512 & 23 \\
Total & 336,641 & 130,487 & & 481,672 & 118,064 \\
\hline
\end{tabular}




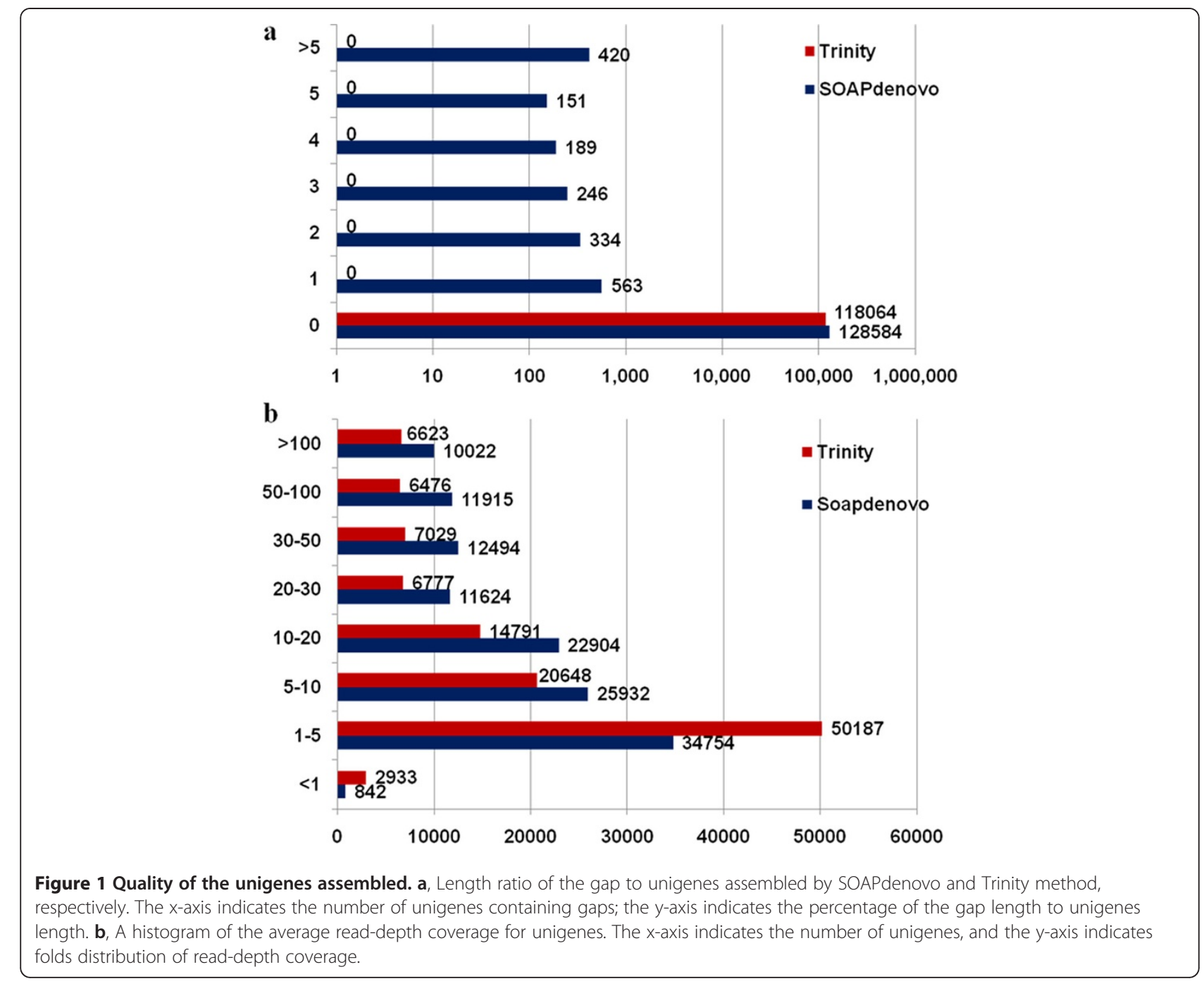

\section{Annotation and classification of the root transcriptome in} ae. Variabilis

To validate and annotate the assembled unigenes, the 118,064 unigenes generated by Trinity were subjected to BLASTX searches (E-value $\leq 1 \mathrm{e}-5)$ against public protein databases. As a result, 72,170 (61.13\%), 52,630 (44.58\%), and 37,993 (32.18\%) unigenes had homologous sequences in NR, Swiss-Prot, and KEGG databases, respectively (Figure 2). Among the unigenes, 50,336 (42.63\%) were synchronously annotated by NR and Swiss-Prot, 37,630 (31.87\%) by NR and KEGG, and 35,379 (29.97\%) by SwissProt and KEGG, and 35,259 (29.86\%) unigenes were simultaneously annotated by all three databases. Also, 43,357 (36.72\%) unigenes showed no homology to known sequences deposited in these databases (Figure 2).

\section{GO classifications}

The unigenes homologous to known sequences in NR, Swiss Prot, and KEGG were further annotated with GO terms using Blast2GO [39]. A total of 31,789 (26.93\%) unigenes were assigned 141,172 GO term annotations, which could be classified into three categories; biological process, molecular function, and cellular component. The biological process category consisted of 42,509 GO terms, which were assigned to 17,953 (15.21\%) unigenes. The molecular function category consisted of 70,401 GO terms, which were assigned to 23,381 (19.80\%) unigenes, and the cellular component category consisted of 28,262 GO terms, which were assigned to 23,798 (20.16\%) unigenes. In addition, 12,340 (10.45\%) unigenes were simultaneously annotated in all three categories (Figure 3). Within the biological process category, unigenes were assigned to "metabolic process" (11,659 terms), "cellular process" (10,593 terms), "response to stimulus" (3,121 terms), "localization" (2,716 terms), and "establishment of localization" (2,487 terms). In the cellular component category, most unigenes were assigned to "cell" (23,598 terms), "cell part" (21,720 terms), and "organelle" (18,647 terms). In the molecular function category, the major GO terms were "catalytic activity" (13,251 terms) and "binding" (12,728 terms). 


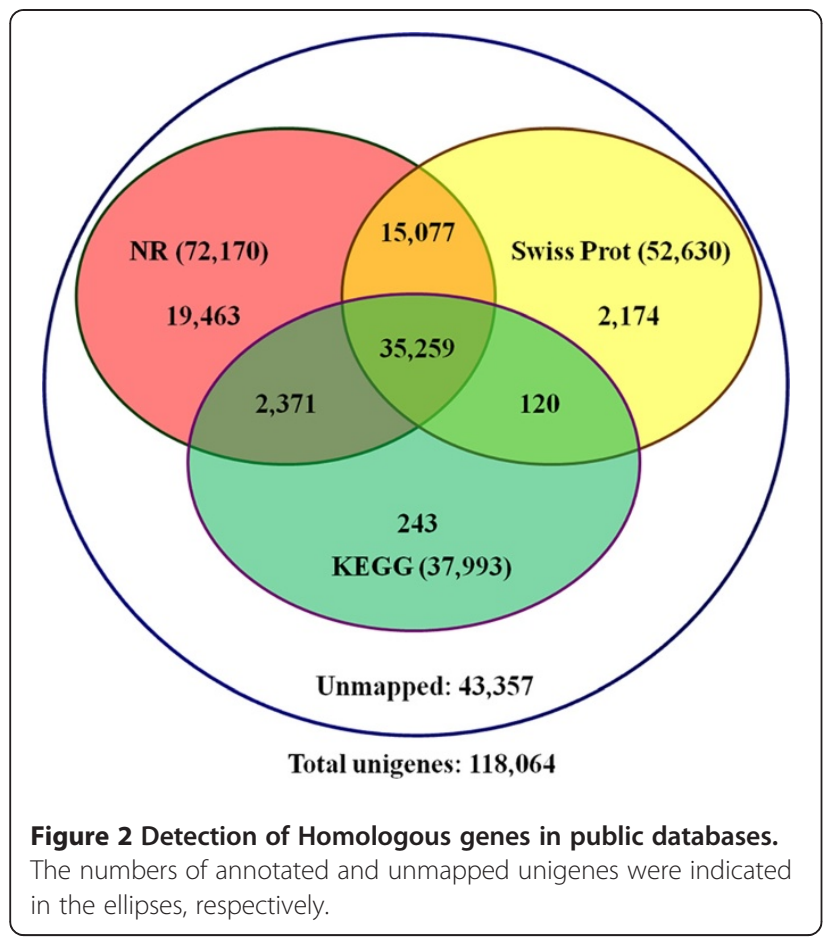

The five subcategories, "response to stimulus", "death", "immune system process", "cell killing" and "antioxidant activity", are all involved in resistance-related biological processes in the responses to abiotic and biotic stimulus/stress, based on their function explanations (Additional file 2).

\section{KEGG pathway mapping}

To identify biological pathways activated in the root of Ae. variabilis, the assembled unigenes were annotated with Enzyme Commission (EC) numbers from BLASTX alignments against the KEGG database (E-value $\leq 1 \mathrm{e}-5)$. The assigned EC numbers were subsequently mapped to the reference canonical pathways. As a result, 37,993 unigenes (32.18\% of 118,064) matched 57,975 members involved in 119 KEGG pathways (Additional file 3). Of the 37,993 unigenes, 9,596 were related to metabolic pathways, 4,815 to biosynthesis of secondary metabolites, 3,355 to spliceosome, 3,216 to plant-pathogen interaction, and 2,275 to ribosome.

Furthermore, 3,798 (including 3,712 individual unigenes) of the 57,975 members were sorted into the plant immune response pathways category, which includes plant-pathogen interaction, phosphatidylinositol signaling system, and $\mathrm{ABC}$ transporters (Additional file 3). These pathways are closely related to plant defense against biotic/abiotic stress.

\section{COG classification}

All assembled unigenes were further annotated based on COG category [46]. A total of 28,126 unigenes were assigned 64,441 functional annotations, which could be grouped into 25 functional categories (Figure 4). The largest category was "General function prediction only" (7,888 COG annotations, $12.24 \%$ of 64,441$)$. Approximately $36.5 \%$ of the COG categories were associated with

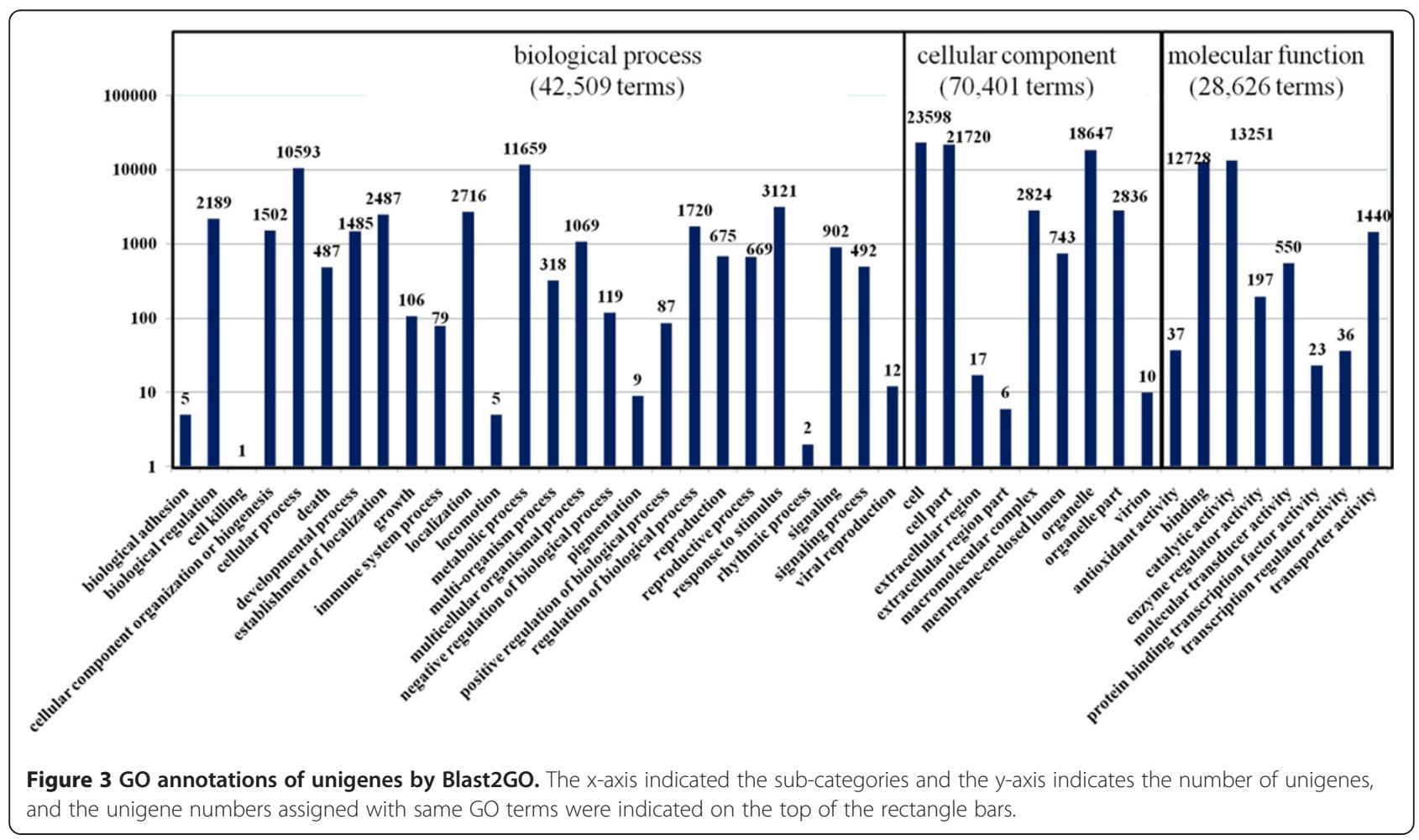




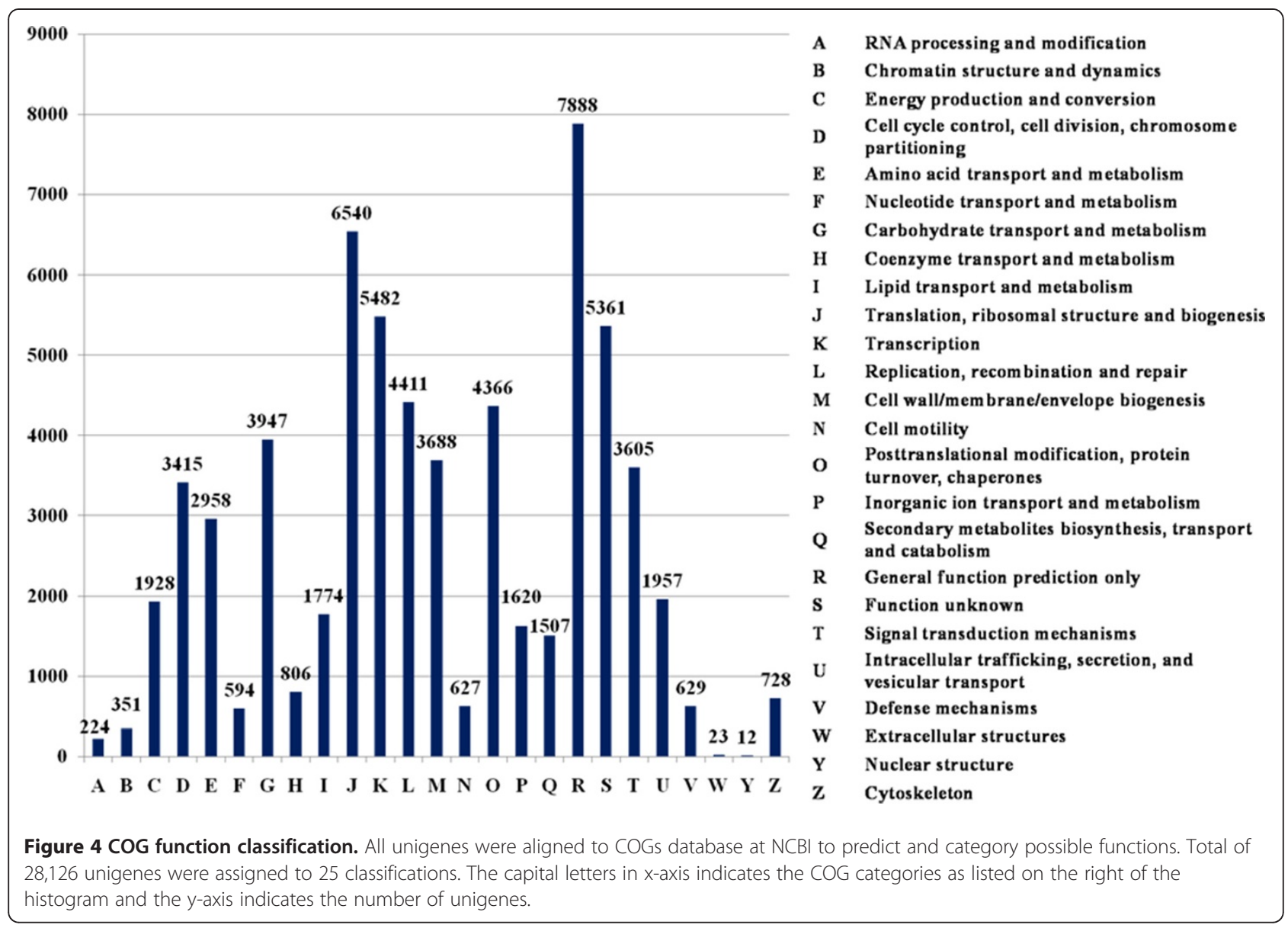

root development, including "Translation, ribosomal structure and biogenesis" (6,540, 10.15\%), "Transcription" (5,482, 8.51\%), "Posttranslational modification, protein turnover, chaperones" $(4,366,6.78 \%)$, “Cell wall/membrane/envelope biogenesis" (3,688, 5.72\%) and "Cell cycle control, cell division, chromosome partitioning" (3,415, $5.30 \%)$, etc. In addition, 5,361 (8.32\%) unigenes belonged to the "Function unknown" category.

The category of "Defense mechanisms" (629, 0.98\%) is closely related to plant defense. The most abundant type of sequence in this category was ABC-type multidrug transport system [47]. A total of 486 unigenes belonged to ATPase and permease (Additional file).

In summary, $78,467(66.46 \%$ of 118,064$)$ unigenes were annotated in the four public databases. Among them, 74,707 unique unigenes were annotated in NR, Swiss-Prot, and KEGG databases through sequence-based alignments (Figure 2). Further, 22,522 unigenes were annotated in the COG database via domain-based alignments (Figure 3), which provided a further 3,760 annotated unigenes. Approximately one-quarter $(18,762)$ of the annotated unigenes were simultaneously annotated with defined functional annotations in the four public databases.
Their functional assignments are summarized in Additional file 5 .

Among the annotated unigenes, $839(0.17 \%$ of 118,064$)$ showed high homology to sequences of nematode species, e.g. Caenorhabditis elegans, Brugia malayi, and Globodera rostochiensis, etc. (Additional file 6).

\section{Expression level}

Gene expression levels were estimated by RPKM values. The distribution of RPKM values indicated that most genes were expressed at low levels. Among 118,064 unigenes, $31,484(26.67 \%)$ had RPKM values of less than 1 , and 96,687 (81.89\%) had RPKM values of less than 10 . The RPKM values of 2152 unigenes (1.82\%) were greater than 100 (Additional file 5).

\section{Discussion}

Ae. variabilis accession No.1 is a valuable resource for development of CCN-resistance in wheat breeding $[12,13]$. However, it is difficult to screen for genes associated with $\mathrm{CCN}$ resistance when genomic information is not available. Transcriptomic profiling provides abundant information for a wide range of biological studies. Transcriptomic 
data gives fundamental insights into biological processes. It can reveal gene expression profiles after experimental treatments or infection, and analyses of conserved orthologous genes can be used for phylogenomic purposes, etc. [48]. Here, we used high-throughput deep sequencing technology to profile the root transcriptome of Ae. variabilis using the Illumina HiSeq 2000 platform. To the best of our knowledge, this is the first report on this subject for Ae. variabilis. The cDNA library was constructed using pooled RNA samples from $\mathrm{CCN}$-infected and non-infected plants at three time points. This maximized the number of expressed transcripts included in the analysis, especially those related to $\mathrm{CCN}$ resistance.

Accurate sequencing and reliable read assembly are essential for downstream applications of transcriptome data [49]. In this study, we used two popular assemblers, SOAPdenovo and Trinity, for de novo assembly of the transcriptomic data of Ae. variabilis. The SOAPdenovo program has been widely used in many studies [25,50], while the Trinity method is a newly developed tool. Trinity was reported to recover more full-length transcripts across a broad range of expression levels, and to provide a unified, sensitive solution for transcriptome reconstruction in species without a reference genome, similar to methods that rely on genome alignments [19]. The two methods showed similar average read-depth coverage values. SOAPdenovo produced more unigenes than Trinity; however, many of the sequences assembled by SOAPdenovo were shorter than 200 bp $(37,828$ out of 130,487$)$. On the other hand, Trinity generated 118,064 unigenes, the unigenes did not contain gaps, and the average unigene length was nearly twice that of those produced by SOAPdenovo (mean length of 599 bp using Trinity, 351 bp using SOAPdenovo). Therefore, Trinity was a better approach than SOAPdenovo for assembly in this research.

The Roche 454 GS FLX platform produces long reads ( $>400 \mathrm{bp}$ ), whereas the Illumina sequencer generates more reads with a shorter length $(90 \mathrm{bp})$. In this study, however, most of the assembled unigenes (130,487 from SOAP denovo ( $\geq 150 \mathrm{bp})$ or 118,064 from Trinity ( $\geq 200 \mathrm{bp})$ ) achieved a higher coverage of $\sim 33 \times$. This indicates that short-read sequencing combined with an in-depth sequencing strategy and an effective assembly tool is an appropriate strategy to analyze transcriptome profiles.

Compared with other transcriptome studies, the length distribution of the 130,487 and 118,064 unigenes generated in this work tended towards shorter-length reads. There are several possible explanations for this. First, Ae. variabilis $\left(2 \mathrm{n}=4 \mathrm{x}=28\right.$, UUS $\left.S^{\mathrm{v}}\right)$ is an allotetraploid species of the tribe Titiceae and it has an enormously expanded repeated genome. This may present a substantial barrier to assembling short unigenes into long ones using current and upcoming sequencing technology $[51,52]$. Second, the total RNA for sequencing in our work was pooled from six samples, which may negatively affect read assembly [53]. The high dynamic range of mRNA expression is a problem for comprehensive de novo mRNA sequencing and assembly [50]. Third, high frequencies of alternative splicing and fusion events may have restricted the assembly of short sequences into longer ones [54,55]. Another important reason is that more than $80 \%$ of unigenes in this study were expressed at low levels. Therefore, there would be fewer reads corresponding to these unigenes for sequencing and for use in sequence assembly. Even so, the de novo transcriptome of Ae. variabilis provided abundant unigene information without gaps in sequences. This genetic data enriches the genomic resources for the tribe Titiceae.

A total of 7,408 individual unigenes $(6.27 \%$ of 118,064$)$ were associated with plant defense and resistance (Additional file 7). These unigenes could be classified into five GO sub-categories, three pathways, and a COG function group. More attention should be paid to the three pathways related to plant defense, which included 3712 unigenes. In the "plant-pathogen interaction" pathway, unigenes were mainly involved with the hypersensitive response, cell wall reinforcement, stomatal closure, and defense-related gene induction (Additional file 8). In the "phosphatidylinositol signaling system" pathway, unigenes were mainly related to reactions involving phosphatidylinositol and its derivatives (Additional file 9). In the "ABC transporters" pathway, unigenes were related to eukaryotic-type transporters only, such as the ABCA subfamily, ABCB subfamily, ABCC subfamily, ABCG subfamily, and other putative $\mathrm{ABC}$ transporters (Additional file 10). These pathways provide a starting point to explore the genes related to $\mathrm{CCN}$ resistance and to understand its molecular mechanism.

Interestingly, 839 unigenes showed high homology to genes from nematode species (Additional file 6), probably because the root had been invaded by CCNs. As there is no genomic information available for $\mathrm{CCN}$, we cannot thoroughly filter sequences of $H$. avenae genes from the transcriptome database. However, the detection of $\mathrm{CCN}$ unigenes confirmed that the method used for $\mathrm{CCN}$ inoculation was successful. More importantly, these unigenes represent those expressed during the interaction with a resistant host. Therefore, this experimental system and the unigene dataset obtained from it build a platform for combining genetic, genomic, and expression information on the interaction between $\mathrm{CCN}$ and its host in future studies [56].

\section{Conclusions}

This is the first report of transcriptome profiling of Ae. variabilis using high-throughput deep sequencing technology. The sequencing was at a depth of 4.69 gigabase pairs. A total of 118,064 unigenes were assembled and 78,467 unigenes were functionally annotated. By including RNA 
samples from $\mathrm{CCN}$-infected plants, the dataset shown here may reveal important information about gene expression related to the plant response to, and defense against, $\mathrm{CCN}$ invasion. Consequently, the large number of transcriptomic sequences and their functional annotations will provide sufficient information to discover novel genes and to explore the molecular mechanism of $\mathrm{CCN}$ resistance in Ae. variabilis. Therefore, the results of this study will be useful for improving CCN resistance in wheat breeding programs.

\section{Additional files}

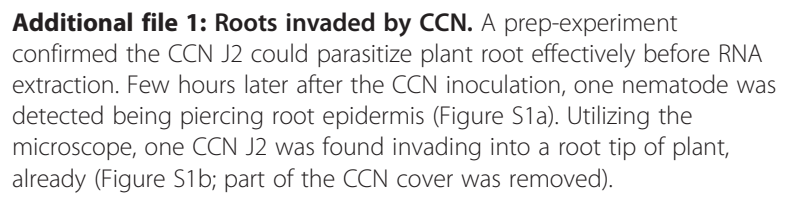

Additional file 2: Resistance related unigenes from GO classification CCN.

Additional file 3: KEGG pathway mapping.

Additional file 4: Defending unigenes from COG alignment.

Additional file 5: Unigene annotations in public databases.

Additional file 6: Nematode-like unigenes list in the transcriptome database.

Additional file 7: Resistance candidate unigenes in this study. Additional file 8: Unigenes involved in plant-pathogen interaction pathway.

Additional file 9: Unigenes involved in phosphatidy linositol signaling system pathway.

Additional file 10: Unigenes involved in $A B C$ transporters pathway.

\section{Competing interests}

The authors declare that they have no competing interests.

\section{Acknowledgements}

We thank Associate Prof. Feng Liu of ShanDong Agricultural University for CCN supply, Associate Prof. Wen-Kun Huang of Chinese Academy of Agricultural Sciences for technique aiding in CCN inoculation, Prof. XiangZhen $\mathrm{Li}$, CIB of CAS, for kindly suggestions on the manuscript. We also thank for the patience of Editor Board and constructive comments of the anonymous reviewers to improve our manuscript. This work was supported by the National Natural Science Foundation of China (30971903) and the National S\&T Key Project of China on GMO Cultivation for New Varieties (2008ZX08009-003).

\section{Author details}

${ }^{1}$ Chengdu Institute of Biology, Chinese Academy of Sciences, Chengdu, Sichuan, China. ${ }^{2}$ Graduate University of the Chinese Academy of Sciences, Beijing, China.

\section{Authors' contributions}

MQY and $H L$ conceived this study. DLX and $\mathrm{HL}$ designed the experimental plan, drafted and revised the manuscript. DLX, HL, JJL, JZ, XC, JLL, ZFP and GBD participated in sample collection, RNA preparation, performed experiments, analyzed and interpreted the sequence data. All authors read and approved the final manuscript.

Received: 15 August 2011 Accepted: 11 April 2012

Published: 11 April 2012

\section{References}

1. Holgado R, Andersson S, Magnusson C: Management of cereal cyst nematodes, Heterodera spp., in norway. Comm Appl Biol Sci Ghent University 2006, 71(3a):7.
2. Williamson V, Kumar A: Nematode resistance in plants: the battle underground. Trends in Genet 2006, 22(7):396-403.

3. Sharma SN, Sain RS, Mathur BN, Sharma GL, Bhatnagar VK, Singh H, Midha RL: Development and validation of the first cereal cyst nematode (Heterodera avenae) resistant wheat CCNRV 1 for northern India. SABRAO J Breeding and Genet 2007, 39(1):1-16.

4. Bonfil DJ, Dolgin B, Mufradi I, Asido S: Bioassay to Forecast Cereal Cyst Nematode Damage to Wheat in Fields. Precis Agric 2004, 5(4):329-344.

5. Nicol JM, Rivoal R: Global Knowledge And Its Application For The Integrated Control And Management Of Nematodes On Wheat. In Integrated Management and Biocontrol of Vegetable and Grain Crops Nematodes. vol. 2nd edition. Edited by Ciancio A, Mukerji KG.: Springer Netherlands; 2007:251-294.

6. McCarter J: Molecular Approaches Toward Resistance to Plant-Parasitic Nematodes. In Cell Biology of Plant Nematode Parasitism. vol. 15th edition. Edited by Berg R, Taylor C.: Springer Berlin/Heidelberg; 2009:239-267.

7. Smiley RW, Nicol JM: Nematodes which Challenge Global Wheat Production. In: Wheat Science and Trade. Wiley-Blackwell; 2009:171-187.

8. Bakker E, Dees R, Bakker J, Goverse A: Mechanisms Involved in Plant Resistance to Nematodes. In: Multigenic and Induced Systemic Resistance in Plants. Edited by Tuzun S, Bent E: Springer US; 2006: 314-334.

9. Coriton O, Barloy D, Huteau V, Lemoine J, Tanguy A-M, Jahier J: Assignment of Aegilops variabilis Eig chromosomes and translocations carrying resistance to nematodes in wheat. Genome 2009, 52(4):338-346.

10. Spetsov P, Mingeot D, Jacquemin JM, Samardjieva K, Marinova E: Transfer of powdery mildew resistance from Aegilops variabilis into bread wheat. Euphytica 1997, 93(1):49-54.

11. Mujeeb-Kaz A, Gul A, Farooq M, Rizwan S, Mirza Jl: Genetic diversity of Aegilops variabilis ( $2 n=4 x=28$; UUSS) for wheat improvement: morphocytogenetic characterization of some derived amphiploids and their practical significance. Pak J Bot 2007, 39(1):57-66.

12. Person-Dedryver F, Jahier J: Cereals as hosts of Meloidogyne naasi Franklin. III. Investigations into the level of resistance of wheat relatives. Agronomie 1985, 5(7):6.

13. Barloy D, Lemoine J, Abelard P, Tanguy A, Rivoal R, Jahier J: Marker-assisted pyramiding of two cereal cyst nematode resistance genes from Aegilops variabilis in wheat. Mol Breed 2007, 20(1):31-40.

14. Kilian B, Mammen K, Millet E, Sharma R, Graner A, Salamini F, Hammer K, Özkan H: Aegilops. In: Wild Crop Relatives: Genomic and Breeding Resources. Edited by Kole C: Springer Berlin Heidelberg; 2011: 1-76.

15. González-Ballester D, Casero D, Cokus S, Pellegrini M, Merchant SS, Grossman AR: RNA-Seq Analysis of Sulfur-Deprived Chlamydomonas Cells Reveals Aspects of Acclimation Critical for Cell Survival. Plant Cell 2010, 22 (6):2058-2084

16. Lee S, Seo CH, Lim B, Yang JO, Oh J, Kim M, Lee S, Lee B, Kang C, Lee S: Accurate quantification of transcriptome from RNA-Seq data by effective length normalization. Nucleic Acids Res 2011, 39(2):e9.

17. Wang Z, Gerstein M, Snyder M: RNA-Seq: a revolutionary tool for transcriptomics. Nat Rev Genet 2009, 10(1):57-63.

18. Mortazavi A, Williams BA, McCue K, Schaeffer L, Wold B: Mapping and quantifying mammalian transcriptomes by RNA-Seq. Nat Meth 2008, 5 (7):621-628.

19. Grabherr MG, Haas BJ, Yassour M, Levin JZ, Thompson DA, Amit I, Adiconis X, Fan L, Raychowdhury R, Zeng $Q$ et al: Full-length transcriptome assembly from RNA-Seq data without a reference genome. Nat Biotechnol 2011, 29:644-652.

20. Vera JC, Wheat CW, Fescemyer HW, Frilander MJ, Crawford DL, Hanski I, Marden $\mathrm{JH}$ : Rapid transcriptome characterization for a nonmodel organism using 454 pyrosequencing. Mol Ecol 2008, 17(7):1636-1647.

21. Meyer E, Aglyamova G, Wang S, Buchanan-Carter J, Abrego D, Colbourne J, Willis $B$, Matz M: Sequencing and de novo analysis of a coral larval transcriptome using 454 GSFIx. BMC Genomics 2009, 10(1):219.

22. Kristiansson E, Asker N, Forlin L, Larsson DJ: Characterization of the Zoarces viviparus liver transcriptome using massively parallel pyrosequencing. BMC Genomics 2009, 10(1):345.

23. Graham IA, Besser K, Blumer S, Branigan CA, Czechowski T, Elias L, Guterman I, Harvey D, Isaac PG, Khan AM, et al: The Genetic Map of Artemisia annua L. Identifies Loci Affecting Yield of the Antimalarial Drug Artemisinin. Science 2010, 327(5963):328-331.

24. Wang Z, Fang B, Chen J, Zhang X, Luo Z, Huang L, Chen X, Li Y: De novo assembly and characterization of root transcriptome using Illumina 
paired-end sequencing and development of cSSR markers in sweetpotato (Ipomoea batatas). BMC Genomics 2010, 11(1):726

25. Shi C-Y, Yang H, Wei C-L, Yu O, Zhang Z-Z, Jiang C-J, Sun J, Li Y-Y, Chen Q, $X i a T$, et al: Deep sequencing of the Camellia sinensis transcriptome revealed candidate genes for major metabolic pathways of tea-specific compounds. BMC Genomics 2011, 12(1):131.

26. Natarajan P, Parani M: De novo assembly and transcriptome analysis of five major tissues of Jatropha curcas L using GS FLX titanium platform of 454 pyrosequencing. BMC Genomics 2011, 12(1):191.

27. Seah S, Miller C, Sivasithamparam K, Lagudah ES: Root responses to cereal cyst nematode (Heterodera avenae) in hosts with different resistance genes. New Phytol 2000, 146(3):527-533.

28. Simonetti E, Alba E, Montes M, Delibes Á, López-Braña I: Analysis of ascorbate peroxidase genes expressed in resistant and susceptible wheat lines infected by the cereal cyst nematode. Heterodera avenae Plant Cell Rep 2010, 29(10):1169-1178.

29. Andres MF, Melillo MT, Delibes A, Romero MD, Bleve-Zacheo T: Changes in wheat root enzymes correlated with resistance to cereal cyst nematodes. New Phytol 2001, 152(2):343-354.

30. Mazarei M, Liu W, Al-Ahmad H, Arelli P, Pantalone V, Stewart C: Gene expression profiling of resistant and susceptible soybean lines infected with soybean cyst nematode. TAG Theoretical and Applied Genetics 2011, 123(7):1193-1206.

31. Williamson VM, Hussey RS: Nematode pathogenesis and resistance in plants. The Plant Cell 1996, 8:11.

32. Das S, Ehlers JD, Close TJ, Roberts PA: Transcriptional profiling of root-knot nematode induced feeding sites in cowpea (Vigna unguiculata L. Walp) using a soybean genome array. BMC Genomics 2010, 11(1):480.

33. Peng $X$, Wood C, Blalock E, Chen K, Landfield P, Stromberg A: Statistical implications of pooling RNA samples for microarray experiments. BMC Bioinforma 2003, 4(1):26.

34. Liu S, Lin L, Jiang P, Wang D, Xing Y: A comparison of RNA-Seq and high-density exon array for detecting differential gene expression between closely related species. Nucleic Acids Res 2010, 39(2):578-588.

35. Everett MV, Grau ED, Seeb JE: Short reads and nonmodel species: exploring the complexities of next-generation sequence assembly and SNP discovery in the absence of a reference genome. Mol Ecol Resour 2011, 11:93-108.

36. Li R, Zhu H, Ruan J, Qian W, Fang X, Shi Z, Li Y, Li S, Shan G, Kristiansen K, et al: De novo assembly of human genomes with massively parallel short read sequencing. Genome Res 2009, 20(2):265-272.

37. Iseli $C$, Jongeneel CV, Bucher P: ESTScan: a program for detecting, evaluating, and reconstructing potential coding regions in EST sequences. Proceedings/International Conference on Intelligent Systems for Molecular Biology; ISMB International Conference on Intelligent Systems for Molecular Biology 1999:138-148.

38. Robinson $M$, Oshlack A: A scaling normalization method for differential expression analysis of RNA-seq data. Genome Biol 2010, 11(3):R25.

39. Conesa A, Götz S, García-Gómez JM, Terol J, Talón M, Robles M: Blast2GO: a universal tool for annotation, visualization and analysis in functional genomics research. Bioinformatics 2005, 21(18):3674-3676.

40. Botstein D, Cherry JM, Ashburner M, Ball CA, Blake JA, Butler H, Davis AP, Dolinski K, Dwight SS, Eppig JT, et al: Gene Ontology: tool for the unification of biology. Nat Genet 2000, 25(1):25-29.

41. Kanehisa M, Goto S: KEGG: Kyoto Encyclopedia of Genes and Genomes. Nucleic Acids Res 2000, 28(1):27-30.

42. Rismani-Yazdi H, Haznedaroglu B, Bibby K, Peccia J: Transcriptome sequencing and annotation of the microalgae Dunaliella tertiolecta: Pathway description and gene discovery for production of nextgeneration biofuels. BMC Genomics 2011, 12(1):148.

43. Moriya $Y$, Itoh M, Okuda S, Yoshizawa AC, Kanehisa M: KAAS: an automatic genome annotation and pathway reconstruction server. Nucleic Acids Res 2007, 35(suppl 2):W182-W185.

44. Mao X, Cai T, Olyarchuk JG, Wei L: Automated genome annotation and pathway identification using the KEGG Orthology (KO) as a controlled vocabulary. Bioinformatics 2005, 21(19):3787-3793.

45. Wall PK, Leebens-Mack J, Chanderbali A, Barakat A, Wolcott E, Liang H, Landherr L, Tomsho L, Hu Y, Carlson J, et al: Comparison of next generation sequencing technologies for transcriptome characterization. BMC Genomics 2009, 10(1):347.

46. Tatusov RL, Natale DA, Garkavtsev IV, Tatusova TA, Shankavaram UT, Rao BS, Kiryutin B, Galperin MY, Fedorova ND, Koonin EV: Genetic diversity of
Aegilops variabilis for wheat improvement. Nucleic Acids Res 2001, 29(1):7.

47. Peng Y, Abercrombie LLG, Yuan JS, Riggins CW, Sammons RD, Tranel PJ, Stewart CN: Characterization of the horseweed (Conyza canadensis) transcriptome using GS-FLX 454 pyrosequencing and its application for expression analysis of candidate non-target herbicide resistance genes. Pest Manage Sci 2010, 66(10):1053-1062.

48. Surget-Groba Y, Montoya-Burgos Jl: Optimization of de novo transcriptome assembly from next-generation sequencing data. Genome Res 2010, 20(10):1432-1440.

49. Feldmeyer B, Wheat C, Krezdorn N, Rotter B, Pfenninger M: Short read Illumina data for the de novo assembly of a non-model snail species transcriptome (Radix balthica, Basommatophora, Pulmonata), and a comparison of assembler performance. BMC Genomics 2011, 12(1):317.

50. Adamidi C, Wang Y, Gruen D, Mastrobuoni G, You X, Tolle D, Dodt M, Mackowiak SD, Gogol-Doering A, Oenal P, et al: De novo assembly and validation of planaria transcriptome by massive parallel sequencing and shotgun proteomics. Genome Res 2011, 21(7):1193-1200.

51. Ness $R$, Siol M, Barrett $S$ : De novo sequence assembly and characterization of the floral transcriptome in cross- and self-fertilizing plants. BMC Genomics 2011, 12(1):298.

52. Mach J: On the Habits of Transposons: Dissociation Mapping in Maize and Megabase Sequencing in Wheat Reveal Site Preferences, Distribution, and Evolutionary History. The Plant Cell Online 2010, 22 (6):1650-1652.

53. Chen S, Yang P, Jiang F, Wei Y, Ma Z, Kang L: De novo analysis of transcriptome dynamics in the migratory locust during the development of phase traits. PLoS One 2010, 5(12):e15633.

54. Asmann YW, Hossain A, Necela BM, Middha S, Kalari KR, Sun Z, Chai H-S, Williamson DW, Radisky D, Schroth GP et al: A novel bioinformatics pipeline for identification and characterization of fusion transcripts in breast cancer and normal cell lines. Nucleic Acids Research 2011.

55. Maher CA, Palanisamy N, Brenner JC, Cao X, Kalyana-Sundaram S, Luo S, Khrebtukova I, Barrette TR, Grasso C, Yu J, et al: Chimeric transcript discovery by paired-end transcriptome sequencing. Proc Natl Acad Sci 2009, 106(30):12353-12358

56. Jacob J, Mitreva M: Transcriptomes of Plant-Parasitic Nematodes. In: Genomics and Molecular Genetics of Plant-Nematode Interactions. Edited by John Jones GG, Carmen Fenoll: Springer Netherlands; 2011: 119-138.

doi:10.1186/1471-2164-13-133

Cite this article as: Xu et al:: De novo assembly and characterization of the root transcriptome of Aegilops variabilis during an interaction with the cereal cyst nematode. BMC Genomics 2012 13:133.

\section{Submit your next manuscript to BioMed Central and take full advantage of:}

- Convenient online submission

- Thorough peer review

- No space constraints or color figure charges

- Immediate publication on acceptance

- Inclusion in PubMed, CAS, Scopus and Google Scholar

- Research which is freely available for redistribution 